\title{
Neutralizing trapped electrons on the hydrogenated surface of a diamond amplifier
}

\author{
Xiangyun Chang, ${ }^{1}$ Ilan Ben-Zvi, ${ }^{1}$ Triveni Rao, ${ }^{1}$ John Smedley, ${ }^{1}$ Erdong Wang, ${ }^{2}$ Qiong Wu,,${ }^{1}$ and Tianmu Xin ${ }^{3}$ \\ ${ }^{1}$ Brookhaven National Laboratory, Upton, New York 11973, USA \\ ${ }^{2}$ Institute of Heavy Ion Physics, School of Physics, Peking University, Beijing 100081, China \\ ${ }^{3}$ State University of New York at Stony Brook, Stony Brook, New York 11974, USA
}

(Received 18 October 2011; published 20 January 2012)

\begin{abstract}
We discuss our investigation of electron trapping in a diamond amplifier (DA). Our previous work demonstrated that some electrons reaching the DA's hydrogenated surface are not emitted. The state and the removal of these electrons is important for DA applications. We found that these stopped electrons are trapped, and cannot be removed by a strong reversed-polarity electric field; to neutralize this surface charge, holes must be sent to the hydrogenated surface to recombine with the trapped electrons through the Shockley-Read-Hall surface-recombination mechanism. We measured the time taken for such recombination on the hydrogenated surface, viz. the recombination time, as less than $5 \mathrm{~ns}$, limited by the resolution of our test system. With this measurement, we demonstrated that DA could be operated in an rf cavity with frequency of a few hundred megahertz.
\end{abstract}

DOI: 10.1103/PhysRevSTAB.15.013501

PACS numbers: 29.25.Bx

\section{INTRODUCTION}

A diamond amplifier (DA) following an electron source, such as a photocathode, is very effective for generating high peak and average current, high-brightness electron beams [1-3]. Under the conditions of our current test setup, the highest emission gain is 200 , limited by the maximum primary electron energy $(10 \mathrm{keV})$ from our electron gun. The maximum bunch charge is $500 \mathrm{pC} / \mathrm{mm}^{2}$ (at pulse duration of about $20 \mu \mathrm{s}$ ), and the peak current density of the emission is $70 \mu \mathrm{A} / \mathrm{mm}^{2}$, restricted by the maximum current density of the primary electron (a few $\mu \mathrm{A} / \mathrm{mm}^{2}$ ) of our electron gun. We designed a test of the DA in a superconducting rf cavity with a photocathode to measure the limits of these parameters.

In previous experiments, as illustrated in Fig. 1, the diamond sample we used was metal coated on one surface (M surface), and hydrogenated on the other (H surface). The DC primary beam struck the M surface (cathode), while the anode was grounded through an integrated current-measurement circuit; electrons were emitted from the $\mathrm{H}$ surface while negative high-voltage (HV) pulses were applied to the cathode relative to the anode. We found that a fraction of the electrons were stopped at the $\mathrm{H}$ surface during the emission pulses, with the smallest trapping probability of about $30 \%$.

As Fig. 2 illustrates, electrons can be trapped directly by the surface states on the $\mathrm{H}$ surface (trapped electrons), or blocked by the surface potential barrier (blocked electrons). We collectively term these electrons the "stopped

Published by the American Physical Society under the terms of the Creative Commons Attribution 3.0 License. Further distribution of this work must maintain attribution to the author(s) and the published article's title, journal citation, and DOI. electrons." The trapped electrons are hard to move back to the $\mathrm{M}$ surface, i.e., to clean, due to their strong binding to the surface states. Even the blocked electrons can be trapped by the steep potential wall on the $\mathrm{H}$ surface, and may not be removed by a strong electric field. However, the blocked electrons may be converted into trapped electrons quickly. Then, the accumulation of electrons that fail to be emitted leads to a gradual reduction of the diamond's internal field with time during the applied emission pulses. In our earlier experiments [1-3], we neutralized the stopped electrons between the emission pulses (neutralization period), such that every emission pulse started in a state with no trapped charge.

In designing a DA for operating in an rf cavity, it is important to understand the mechanism of $\mathrm{H}$ surface neutralization. If all the stopped electrons were free to drift backwards to the M surface when we reverse the applied field, they automatically would be cleaned by the strong rf field when the phase reverses the field. Otherwise, holes must be sent to the $\mathrm{H}$ surface during the electrondecelerating phase to recombine with the trapped electrons.

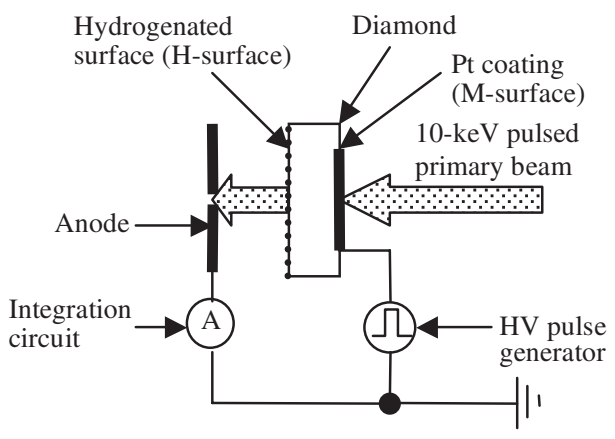

FIG. 1. Diagram of the previous DA emission experiment (see text for explanation). 


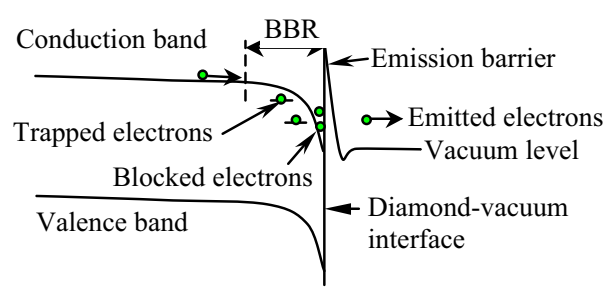

FIG. 2. Possible stopping mechanisms of electrons on the $\mathrm{H}$ surface during emission pulses. This band diagram does not include the effect of the external field.

Furthermore, should neutralization of the $\mathrm{H}$ surface by electron-hole recombination be necessary, the recombination time constant $\left(\tau_{R}\right)$ of a hole with an H-surface-trapped electron becomes an important parameter; a longer $\tau_{R}$ would require more rf cycles than do shorter ones to neutralize the $\mathrm{H}$ surface.

\section{EXPERIMENT}

We conducted the experiments on the same test system we used previously [1-3] with a small modification. As shown in Fig. 3, we moved the HV input from applying negative pulses on cathode to sending positive pulses to the anode. The cathode was grounded through an integrationcurrent measurement circuit; this configuration ensured a constant $10-\mathrm{keV}$ of primary electron energy throughout the experiment. The surface of the diamond sample was metal coated with $30 \mathrm{~nm}$ of $\mathrm{Pt}$ on its primary electron side, and hydrogenated on the emission surface. For hydrogenation, we followed the procedure detailed in [3].

The HV pulses had a rise time and fall time of $20 \mathrm{~ns}$. The HV pulse's width $\left(T_{\mathrm{HV}}\right)$ was fixed to $1 \mu \mathrm{s}$, and the amplitude of the $\mathrm{HV}$ pulse $\left(V_{\mathrm{HV}}\right)$ was adjustable from $0 \mathrm{~V}$ to $5 \mathrm{kV}$; there was a fixed delay between the $\mathrm{HV}$ pulse and its trigger $(220 \pm 5 \mathrm{~ns})$. The HV pulse's frequency was fixed at $1 \mathrm{kHz}$.

The primary electron beam was delivered as either a single or dual pulse per anode HV pulse. The electron gun

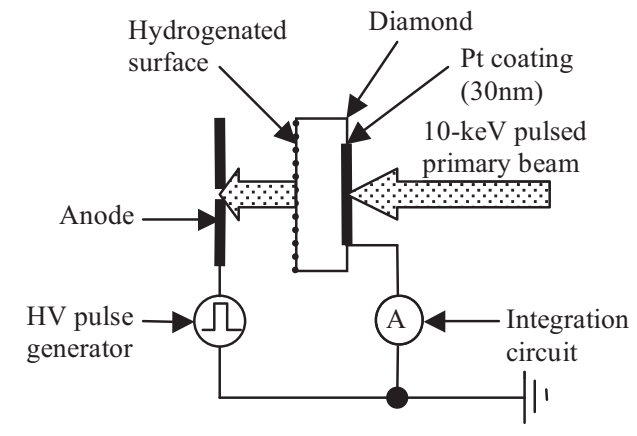

FIG. 3. Diagram of the experimental system. The primary beam's spot was about $1 \mathrm{~mm}^{2}$, the diamond's thickness was $300 \mu \mathrm{m}$, and the gap between the $\mathrm{H}$ surface and the anode was $250 \mu \mathrm{m}$.
(Kimball Physics Inc., EGG-3101) generated the pulsed beam via its grid electrode, so that the primary beam always struck the same spot on the diamond. The rise and fall times of the beam's pulses were less than $5 \mathrm{~ns}$; the beam's pulse widths, $T_{\mathrm{B} 1}$, for the first or a single pulse, and $T_{\mathrm{B} 2}$ for the second pulse, each were from $80 \mathrm{~ns}$ to DC. Similar to the HV pulses, there was a fixed delay between each beam pulse and its trigger (295 $\pm 5 \mathrm{~ns})$. We set the current amplitude of the beam pulses at $300 \mathrm{nA}$.

The average current of cathode $\left(\square_{C}\right)$ was measured through an integrated circuit, $\left|\square_{C}\right|=\left|\square_{E}\right|-\left|\square_{P}\right|$, where $\square_{E}$ was the average emission current, and $\square_{P}$ was the average primary current on the cathode.

Figure 4(a) illustrates the rising and falling edges of the pulses. Beam pulse \#1 was $0.9 \mu$ s earlier than the HV pulse, and was outside it. We termed this the neutralization pulse, because, in the presence of an internal field created by the trapped charge, it generates holes that drift to the $\mathrm{H}$ surface thereby neutralizing the charge. Beam pulse \#2 was swept across the HV pulse from left to right in steps of 10 ns. Two parts of this pulse are distinguishable: One that overlaps a portion of the HV pulse is designated the emission pulse; the rest of beam pulse \#2 is considered part of the neutralization pulse because it functions in the same way as does beam pulse \#1.

Figure 4(b) shows the average emission current at each scanning point. The leftmost vertical line marks the time when beam pulse \#2 starts overlapping the HV pulse, thus enabling emission. The second line marks the beginning (set by the trigger time of the beam's pulses) of when beam pulse \#2 completely overlaps the HV pulse; it ends at the third vertical line. By the fourth (rightmost) vertical line, beam pulse \#2 no longer overlaps the HV pulse.

Before the first vertical line, both beam pulses fell outside the HV pulse, and $I_{E}$ was zero. Between the first and the second vertical line, the emission pulse and $I_{E}$ increased linearly. Between the second and the third vertical line, beam \#2 was embedded completely in the HV pulse, and $I_{E}$ was a constant. Between the third and the fourth vertical line, the emission pulse decreased linearly, as did

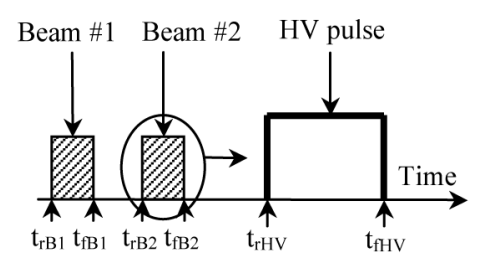

(a)

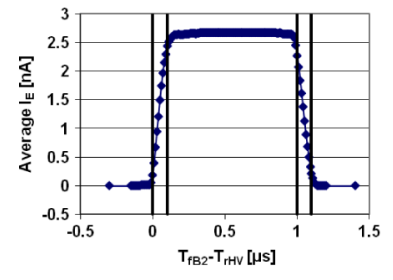

(b)
FIG. 4. (a) Pulsing diagram of the test, $T_{\mathrm{B} 1}=T_{\mathrm{B} 2}=0.1 \mu \mathrm{s}$, $T_{\mathrm{HV}}=1 \mu \mathrm{s}, \quad V_{\mathrm{HV}}=1 \mathrm{kV} ; \quad t_{\mathrm{rHV}}-t_{\mathrm{rB} 1}=0.9 \mu \mathrm{s} ;$ beam \#2 swept across the $\mathrm{HV}$ pulse in steps of $10 \mathrm{~ns}$. (b) Average emission current at each step. First vertical line: $t_{\mathrm{fB} 2}=t_{\mathrm{rHV}}$; second vertical line: $t_{\mathrm{rB} 2}=t_{\mathrm{rHV}}$; third vertical line: $t_{\mathrm{fB} 2}=t_{\mathrm{fHV}}$; fourth vertical line: $t_{\mathrm{rB} 2}=t_{\mathrm{fHV}}$. The emission gain at the flattop was 90 . 
$I_{E}$. Finally, after the fourth vertical line, the beam pulses again fell outside the HV pulse, and $I_{E}$ again was zero.

The emission curve is rounded near the corners of the vertical lines, for example, within $\sim 20 \mathrm{~ns}$ before the first vertical line and after the second one, reflecting the rise and fall time of the HV pulses.

The curve in Fig. 4(b) proves first that an overlap of the primary beam and HV pulse is necessary for electron emission from the DA, and second, that the emission current is independent of the relative position of the emission and HV pulses.

Figure 5 illustrates the results of the measurement with a single-pulse beam. Figure 5(a) is a schematic of the pulse's structure; Fig. 5(b) plots the measured average current as a function of relative time between the beam pulse and the high-voltage pulse. The beam was swept across the HV pulse in steps of $10 \mathrm{~ns}$, from first being ahead of the HV pulse, through their overlap, to being later than it. The first vertical line in Fig. 5(b) represents the time when the beam pulse starts overlapping the HV pulse; the second vertical line marks the start of the complete overlap of the beam by the HV pulse; the third vertical line depicts the emergence of the beam's falling edge out from the HV pulse's falling edge; and, the fourth vertical line represents the egression of the beam's rising edge from the HV pulse's falling edge. Similar to Fig. 4, the rounded curves near the corners of the vertical lines reflect the rise and fall times of the $\mathrm{HV}$ pulses.

Let us call the curve between the first and the second vertical line in Fig. 5(b) the left peak, and the curve between the third and the fourth vertical line in Fig. 5(b) the right peak. The electron beam's pulse is partially outside the HV pulse (neutralization pulse) and partially overlapped by the HV pulse (emission pulse) in both left and right peaks. In the left peak, the width of the emission pulse increases linearly, while the neutralization pulse declines correspondingly as the delay time (horizontal axis) increases linearly within the left peak. Initially, $I_{E}$ increases linearly due to the linear increase of the emission pulse's (a)

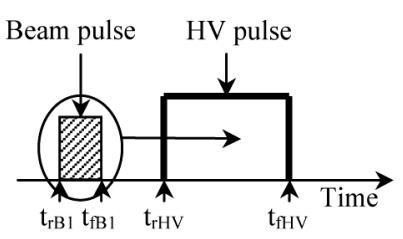

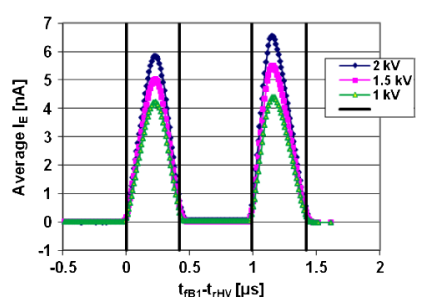

(b)
FIG. 5. (a) Schematic pulse-structure diagram of the test, $T_{\mathrm{B} 1}=0.42 \mu \mathrm{s}, T_{\mathrm{HV}}=1 \mu \mathrm{s}, V_{\mathrm{HV}}=1,1.5,2 \mathrm{kV}$; the beam pulse was swept across the HV pulse in steps of $10 \mathrm{~ns}$. (b) Average $I_{E}$ at each step. The first vertical line: $t_{\mathrm{fB} 1}=t_{\mathrm{rHV}}$; second vertical line: $t_{\mathrm{rB} 1}=t_{\mathrm{rHV}}$; third vertical line: $t_{\mathrm{fB} 1}=t_{\mathrm{fHV}}$; fourth vertical line: $t_{\mathrm{rB} 1}=t_{\mathrm{fHV}}$. width. With the continuing rise in the emission pulse, the emission current, $I_{E}$, begins to fall due to trapping and insufficient neutralization time. This process is reversed in the right peak. When the beam lies completely inside HV pulse (between second and third vertical lines), the neutralization pulse falls to zero, as does $I_{E}$. This finding signified the complete cancellation of the HV pulse's applied field in the diamond, $1.2 \mathrm{MV} / \mathrm{m}$ at $V_{\mathrm{HV}}$ of $2 \mathrm{kV}$, due to the space-charge field of the accumulated stopped electrons on the $\mathrm{H}$ surface; in other words, the trapped electrons could not be removed by this electric field of $1.2 \mathrm{MV} / \mathrm{m}$ in the diamond. We repeated this experiment with a $V_{\mathrm{HV}}$ of $5 \mathrm{kV}$ (not shown in Fig. 5), obtaining the same result.

As shown in Fig. 5(b), the curves are mirror symmetric at $t_{\mathrm{fB} 1}-t_{\mathrm{rHV}}=0.71 \mu \mathrm{s}$ with a small peak-current difference at each side. This small difference in peak current is due to the low drift velocity of the holes from the neutralization pulses. The lower drift velocity of the holes not only reflects the slower mobility of the holes relative to electron mobility, but also the fact that the electric field declines rapidly over the neutralization pulse. In the left peak, the HV pulse sweeps away a portion of the neutralizing holes on the way to reaching the $\mathrm{H}$ surface, thereby shortening the effective length of the beam pulse. However, in the right peak, the holes have a much longer time to accomplish the neutralization.

To measure $\tau_{R}$ more accurately, we completed another dual-pulse experiment (Fig. 6). We embedded beam \#2 (emission pulse) in the HV pulse and beam \#1 (neutralization pulse) approached the HV pulse in steps of $10 \mathrm{~ns}$. As the $I_{E}$ curve in Fig. 6(b) reveals, $I_{E}$ was a constant before beam \#1 moved over the HV pulse, with an error of \pm 5 ns (i.e., the resolution limit of our system), implying that $\tau_{R}$ was smaller than this. The small drop in current before the vertical line was caused by the rising edge of the HV pulse. To measure $\tau_{R}$ with higher resolution, we must test DA in an rf cavity and employ a photocathode primary beam.

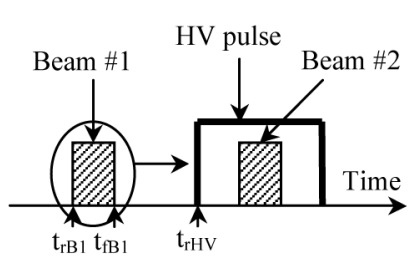

(a)

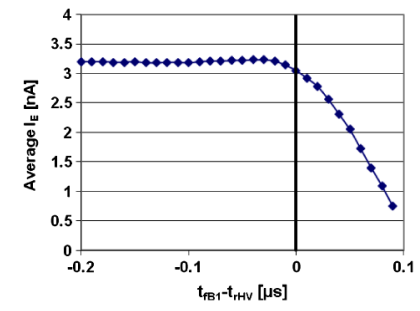

(b)
FIG. 6. (a) Pulsing diagram of the test, $T_{\mathrm{B} 1}=T_{\mathrm{B} 2}=0.1 \mu \mathrm{s}$, $T_{\mathrm{HV}}=1 \mu \mathrm{s}, V_{\mathrm{HV}}=2 \mathrm{kV}$; beam \#2 was embedded in the $\mathrm{HV}$ pulse and beam \#1 approached the HV pulse in steps of $10 \mathrm{~ns}$. (b) Average $I_{E}$ at each step. Vertical line: $t_{\mathrm{fB} 1}=t_{\mathrm{rHV}}$. Peak emission gain was 110 . 


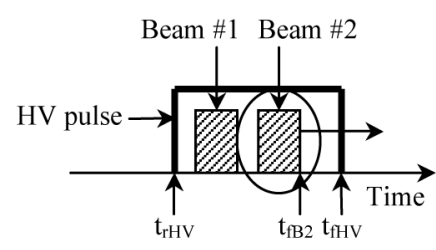

(a)

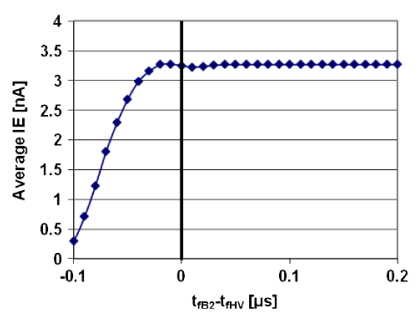

(b)
FIG. 7. (a) Pulsing diagram of the test, $T_{\mathrm{B} 1}=T_{\mathrm{B} 2}=0.1 \mu \mathrm{s}$, $T_{\mathrm{HV}}=1 \mu \mathrm{s}, V_{\mathrm{HV}}=2 \mathrm{kV}$; beam \#1 was embedded in the $\mathrm{HV}$ pulse and beam \#2 swept across its falling edge in steps of $10 \mathrm{~ns}$. (b) Average $I_{E}$ at each step. Vertical line: $t_{\mathrm{rB} 2}=t_{\mathrm{fHV}}$. The peak emission gain was 114 .

In the experiment shown in Fig. 7, beam \#1 (the emission pulse) was embedded in the HV pulse, and beam \#2 was swept across the HV pulse's falling edge in steps of 10 ns. As Fig. 7(b) reveals, $I_{E}$ was a constant after the second beam emerged from the HV pulse. The constant (3.3 nA) is marginally higher than that shown in Fig. 6(b) (3.2 nA). This slight difference may indicate the presence of a minor fraction of trapped electrons with a long recombination time.

\section{DISCUSSION}

In a single-crystal pure diamond, electrons and holes theoretically are forbidden to recombine in the bulk of the diamond due to its indirect band-gap structure [4]. Analogous to $\mathrm{Si}$ with the similar band-gap structure, bulk electron-hole recombination occurs on a millisecond time scale $[5,6]$. Surface recombination is a special case of the Shockley-Read-Hall (SRH) model [7,8], viz. fast trap-assisted recombination. Analogous to the diamond, the SRH time scale on the Si surface is about 200 ps $[9,10]$. As detailed above, most stopped electrons very quickly recombine with holes on the diamond's surface, with $\tau_{R}$ less than $5 \mathrm{~ns}$, denoting that the stopped electrons are dominated by trapped electrons, and they are recombined with the holes through SRH recombination. Our measurement of $\tau_{R}$ on the $\mathrm{H}$ surface agrees with experimental data reported elsewhere [11,12]; the carrier lifetimes on grain boundaries reported therein are less than $1 \mathrm{~ns}$.
Even if $\tau_{R}$ were a few ns, for example $5 \mathrm{~ns}$, a DA still might be operated in rf cavities with a frequency of about $100 \mathrm{MHz}$. The rf frequency would be even higher in those cases where the primary pulse current for detrapping could be greater than that for emission.

\section{CONCLUSIONS}

We experimentally demonstrated that the stopped electrons on the $\mathrm{H}$ surface of a DA during emission pulses were trapped, and could not be removed by a strong electric field (a few $\mathrm{MV} / \mathrm{m}$ in diamond). Holes must be sent to the $H$ surface to recombine with the electrons through SRH recombination. In an $\mathrm{rf}$ cavity, this is achieved by sending primary beams in the decelerating phase. The recombination time of the holes with H-surface-trapped electrons $\left(\tau_{R}\right)$ was below $5 \mathrm{~ns}$, viz. the resolution limit of our test system. Even if $\tau_{R}$ was $5 \mathrm{ns,}$ the DA still could be operated in rf cavities with frequency of more than $100 \mathrm{MHz}$. We expect that $\tau_{R}$ will be much smaller than $5 \mathrm{~ns}$, so that neutralizing the $\mathrm{H}$ surface will not be problematic for rf cavities with a frequency of a few hundred $\mathrm{MHz}$.

[1] X. Chang et al., in Proceedings of the 23rd Particle Accelerator Conference, Vancouver, Canada, 2009, p. 691, TU2GRC03.

[2] X. Chang et al., Phys. Rev. Lett. 105, 164801 (2010).

[3] E. Wang et al., Phys. Rev. ST Accel. Beams 14, 061302 (2011).

[4] W. Saslow, T. K. Bergstresser, and M. L. Cohen, Phys. Rev. Lett. 16, 354 (1966).

[5] B. Mitchell, T. Trupke, J. Weber, and J. Nyhus, J. Appl. Phys. 109, 083111 (2011).

[6] A.V. Zinovchuk and A. K. Tkachenko Fiz. Tekh. Poluprovodn. 45, 62 (2011).

[7] W. Shockley and W. T. Read, Phys. Rev. 87, 835 (1952).

[8] R. N. Hall, Phys. Rev. 87, 387 (1952).

[9] N. Halas and J. Bokor, Phys. Rev. Lett. 62, 1679 (1989).

[10] J. Bokor and R. Storz, Phys. Rev. Lett. 57, 881 (1986).

[11] H. Yoneda et al., J. Appl. Phys. 83, 1730 (1998).

[12] S. Salvatori, M. C. Rossi, and F. Galluzzi, IEEE Trans. Electron Devices 47, 1334 (2000). 